\title{
Analysis of Network Impacts of Frequency Containment provided by Domestic-Scale Devices using Matrix Factorization
}

\author{
Matthew Deakin, Member, IEEE, David M. Greenwood, Member, IEEE, Phil C. Taylor, Senior Member, IEEE, \\ Pete Armstrong, and Sara Walker, Senior Member, IEEE
}

\begin{abstract}
This paper studies the distribution network impacts of frequency containment services derived from domestic-scale devices. Risk metrics considering the likelihood and severity of violations are proposed, given uncertainty in the location of these devices. A novel linearization approach is proposed to enable detailed simulations of large-scale networks, capturing MV-LV coupling in European-style networks of over 100,000 nodes. The approach combines a novel factorization of the power flow Jacobian matrix and an efficient linearization update step. The first of these innovations improves the scalability and practicality of the linearization, reducing the memory and computational requirements by as much as twenty times, whilst the latter reduces the median linearization error by at least $50 \%$ in all networks studied. It is demonstrated that rapid voltage change (RVC) and overvoltage constraints could limit the uptake of these devices to just a fraction of one percent of customers if the response is not managed. Sensitivity analysis demonstrates that both the likelihood and severity of constraint violations increases rapidly with the size of the devices used for frequency response.
\end{abstract}

Index Terms-Frequency response services, unbalanced distribution networks, matrix factorization, probabilistic load flow, MV-LV Systems

\section{INTRODUCTION}

$\mathbf{F}$ REQUENCY response services from distributed energy resources (DERs) have been proposed in many power systems across the world to harness the response available from fleets of domestic devices such as electric vehicles (EVs) or electric hot water tanks. For example, if $1 \%$ of the $27 \mathrm{~m}$ households in Great Britain (GB) had $3 \mathrm{~kW}$ devices for providing frequency response services, $810 \mathrm{MW}$ of response could be delivered, almost half of the $1800 \mathrm{MW}$ largest loss of infeed from 2022 [1]. As such, the UK is presently developing standards for the exploitation of domestic-scale devices for purposes such as this [2]. The impacts of a large fleet of these devices are, however, as-yet unproven in a large power system. Recent events such as the GB 9th August Blackout [3] demonstrate how DERs increasingly affect system security. This uncertainty is compounded by reduced system inertia and increased vulnerability, with the latter caused by new largescale assets such as new nuclear generation and increased HVDC interconnection. When generation-demand imbalance of a power system is in extremis and system frequency is

M. Deakin, D. Greenwood and S. Walker are with Newcastle University, Newcastle-upon-Tyne, Newcastle; P. Taylor is with University of Bristol; P. Armstrong is with Mixergy Ltd. Email: matthew.deakin@newcastle.ac.uk changing rapidly, it is even more important these constraints are maintained, so that frequency- and voltage-sensitive DERs do not trip whilst the frequency is still being stabilised.

There are a wide range of domestic-scale devices that have been proposed for frequency response services, with academic literature typically focusing on ensuring that the fleet's response meets predefined power and energy requirements. Most methods follow early works such as [4], with devices responding to local frequency measurements. Devices proposed for frequency response include heat pumps [5], EVs [6], electric hot water tanks [4], [7], or general thermostatic loads [8]. On the other hand, many recent works consider stochastic hosting capacity of distribution networks (recent works such as [9] even calculate probabilistic hosting capacity for DERs which are actively controlled); these works typically focus on network impacts of DERs during normal operations [10]-[12].

Given that all devices on a network will be responding to the global system frequency signal, there will be little or no temporal diversity in the DER response, leading to significantly increased coupling between LV distribution networks. This necessitates the use of MV-LV models that can capture not only the highly varied voltage drops in LV circuits, but also the coupling that occurs between LV circuits on the MV network [13]-[16].

The closedown report from a GB TSO project on demandside frequency response services [17] concluded that it is vital the industry considers the impact on the distribution network before frequency response from emerging demand side technologies is deployed at scale'. Despite this, there are few works in either industry or academia which study possible distribution network issues caused by this type of control, and those that do focus on slower (secondary) frequency response services. In [18], the authors study the impacts of secondary frequency control provided by thermostatic loads, concluding that network impacts tend to be relatively minor. The same authors subsequently develop a method of controlling thermostatic loads to follow a dynamic frequency regulation signal whilst accounting for steady-state thermal and voltage constraints [19]. Similarly, [20] proposes a method to enable the maximum frequency response whilst respecting network constraints, although only MV level constraints are considered explicitly. Other works such as [21] consider a combined frequency response and voltage-regulation system for an MV microgrid, but only consider impacts of voltage 
in the secondary response. In [22], the authors develop an efficient, iterative method for distributed OPF in large distribution networks; similarly, in [23], the authors propose a framework for responding to TSO instructions, including automatic generation controls (AGC). Communications delays mean that these OPF-based approaches are better suited to slower-acting frequency response. In summary, despite the enormous potential for the provision of fast frequency response services (e.g., frequency containment) from domestic-scale DERs, there is little literature studying the potential network impacts of such devices for customers (i.e., at LV). Such services necessarily lead to very high temporal coupling across a wide geographic area. New methods are therefore required to understand the risks that domestic-scale DERs could pose to power quality when called upon to provide these services, in terms of both the likelihood and severity of constraint violations.

The contribution of this work is to address this gap by proposing a method, based on load flow linearization, for calculating the likelihood and severity of network constraint violations in distribution systems with frequency containment services provided by domestic DERs. The main technical innovation is a new matrix factorization method to enable the linearization approach to scale to MV-LV circuits of over 100,000 nodes, whilst also improving computational performance. In addition, a novel linearization update step exploits the probabilistic formulation, reducing the linearization error with minimal additional computational overhead. Proposed risk metrics are then used to study the hosting capacity of seven MV-LV circuit models, on which sensitivity analysis to DER size and geographic diversity are considered.

The paper is structured as follows. Section II outlines the operation of the domestic devices and the risk metrics considered, to clearly outline the main adverse network impacts of frequency containment services. In Section III, the two innovations are outlined to demonstrate the principles by which they improve computational performance. The results of the case studies are given in Section IV, from which salient conclusions are drawn in Section V.

\section{FREQUENCY CONTAINMENT AND Risk OF Distribution Network CONSTRAint Violations}

This work focuses on events following a sudden loadgeneration imbalance leading to a fast change in frequency, as could happen when an interconnector or nuclear reactor trips. It is assumed that the response from DERs is quick, ramping from zero to rated power in one second or less. This sort of response would be expected of devices providing 'static' or 'dynamic containment' frequency response services (as defined in [24]), in contrast to continuous frequency regulation services which have droop characteristic and lag of several seconds or more. The fastest (subsecond) dynamics of the power system are assumed to have decayed by the point the containment service is activated-the distribution network power flows can therefore reasonably be studied using steadystate load flow analysis. Following the activation of service, it is assumed that the response by domestic devices (or other providers) will typically cease within a few minutes as reserves come online and the system frequency recovers.

A large geographic spread of fast-acting DERs, responding within one second of each other, is unlike other power quality issues that are presently seen in distribution networks [25]. For example, motor transients might last for tens of seconds, but these excursions are limited to a single load bus. At slower timescales, there are correlations between variations in PV generator output, but once geographic diversity is accounted for, ramping typically occurs over several minutes [1].

\section{A. Short-Duration Voltage Constraints}

Three constraints are considered within this work: overvoltage constraints, undervoltage constraints, and rapid voltage change (RVC) constraints. (RVC constraints are called 'voltage deviation' constraints in the US context.) For the $j$ th customer with voltage $V_{j}$, these constraints are represented by the set $\mathcal{S}_{j}$, defined as

$$
\mathcal{S}_{j}=\left\{V_{j}: V_{-}<\left|V_{j}\right|<V_{+} \wedge \Delta\left|V_{j}\right|<\Delta V_{+}\right\},
$$

where $V_{+}, V_{-}, \Delta V_{+}$are the upper, lower, and voltage magnitude change constraints, and the voltage change $\Delta V_{j}$ is given by

$$
\Delta\left|V_{j}\right|=|| V_{j}|-| V_{j}^{\mathrm{Nom} \cdot}||,
$$

where $V_{j}^{\text {Nom. }}$ is the complex voltage prior to the step change in demand. Combining all constraints across each of the loads $j$ gives the network-wide constraint set $\mathcal{S}$ as

$$
\mathcal{S}=\left\{V: V_{j} \in \mathcal{S}_{j} \forall j\right\} .
$$

For the calculations of voltages $V$, the primary substation taps are assumed to not change from the position at which they were set prior to the frequency excursion, as the time constant over which discrete tap operations occur (or that of other regulation equipment such as capacitor banks) can typically be assumed to be over 45 seconds [11].

All three of these constraints are designed to protect devices from malfunctions. As many DERs are sensitive to both voltage and frequency [26], maintaining a strong supply voltage during a large generation-demand imbalance is particularly important. Thermal constraints on distribution network components are not considered within this work, as these components can typically be operated in overloaded conditions for short periods of time on an occasional basis [27].

1) Short-Duration and Rapid Voltage Changes in Network Standards: RMS voltage constraints in most jurisdictions are typically considered over 10 minutes (or similar length), whilst voltage sag, swell and ramping constraints typically consider voltages at short time periods of just a few seconds or less [28], [29]. There appears to therefore be little guidance for voltage sags or swells lasting several minutes. In the GB system, 10minute rms voltages should remain between 0.85 and $1.10 \mathrm{pu}$ $100 \%$ of the time [29]; on the other hand, the 'ITI Curve' [25, Ch. 2] suggests that, over time periods longer than a few seconds, voltages should remain between 0.87 and $1.10 \mathrm{pu}$. For the purposes of this work, conservative bounds of $(0.87$, 1.1) pu are considered to account for the vulnerability of power systems during these events. 
In both the UK and US, RVC constraints following a stepchange in voltages are 3\% [30], [31]. If, instead of changing instantaneously the voltage ramps over a duration of longer than $0.8 \mathrm{~s}$, then there are a range of criteria which must be considered to assess if the power quality is acceptable (e.g., whether the voltage increases or decreases, the number of events per year). In all cases, however, at steady-state the voltage should not have changed more than 3\% [30].

\section{B. Indices for Evaluating Risk of Violations}

In this work the main uncertainty considered is in terms of the location of devices with frequency response capabilities, which are spread throughout the network with uniform probability. Each load is allocated a device of size $P_{0}$ with probability given by the DER penetration $\rho$, so the change in injections at load $j, \Delta P_{j}$, follows a Bernoulli distribution as

$$
\frac{\Delta P_{j}}{P_{0}} \sim \operatorname{Bernoulli}(\rho) .
$$

By (4), DERs are equally likely to be allocated at any customer's premises.

It is assumed that the mostly likely operation of devices is with an on-off characteristic-that is, devices respond if the global frequency crosses a given threshold (as described in, e.g. [32]). Such devices could be maintained by an entity such as an aggregator, and could either have a means of local frequency measurement or reliable, fast communication infrastructure to an external frequency measurement point. For the purposes of this work, all devices are assumed to operate with unity power factor, a reasonable assumption for power electronic-interfaced devices (such as EV chargers) or resistive immersion heaters. The aggregator would take responsibility for ensuring suitable availability and response from the fleet of devices [7], [18].

The study of risk and hosting capacity requires the determination of suitable risk indices [12]. We have developed two risk metrics to capture the likelihood and severity of potential violations in supply quality. The first, referred to as the Likelihood of Voltage Violations (LoVV), is given by

$$
\mathrm{LoVV}=\mathbb{P}(V \notin \mathcal{S}),
$$

where notation $\mathbb{P}(\alpha)$ denotes the probability of event $\alpha$ occurring. This measure determines the likelihood of there being a constraint violation anywhere in the network [31]. As such, it is from the LoVV that the $\zeta_{\%}$ Stochastic Hosting Capacity $\left(\zeta_{\%}\right.$ SHC) value can be determined. That is,

$$
\zeta_{\%} \mathrm{SHC}=\inf \left\{\rho: \operatorname{LoVV}=\zeta_{\%}\right\} .
$$

For example, the 5\% SHC value is the value of $\rho$ for which there will be a $5 \%$ chance of a voltage violation [10].

The second dimension of risk (severity) is captured in this work with the proposed Expected Customer Voltage Violations (ECVV), defined for a network of $N_{\text {Cust }}$ customers as

$$
\mathrm{ECVV}=\mathbb{E}\left(\sum_{j=0}^{N_{\mathrm{Cust}}-1} \frac{1-\mathcal{I}_{S_{j}}\left(V_{j}\right)}{N_{\mathrm{Cust}}}\right),
$$

where $\mathbb{E}$ is the expectation operator, and $\mathcal{I}_{\mathcal{S}_{j}}$ is the indicator function (return 1 if the argument is within $S_{j}$ and zero otherwise). Intuitively, the ECVV defines the expected number of voltage violations for a given DER penetration $\rho$.

To estimate the values of both the ECVV and the LoVV, we use a 'plug-in estimator' [33, Ch. 8], estimating the values of these indices by sampling from (4) and then calculating estimates directly from the load flow solutions from (5), (7). Estimated $\zeta_{\%}$ SHC values are found by linear interpolation from the estimated LoVV. For estimating these values, 3000 Monte Carlo runs are considered for each value of DER penetration $\rho$. This relatively high number of runs (compared to, say, [10]) is required as the DER penetration $\rho$ will be studied with penetrations levels well under $1 \%$, necessitating more Monte Carlo runs.

1) Loading Conditions: To understand the constraints, we use the approach outlined in [34], considering if the DERs cause violations at just two demand conditions, High demand and Low demand. These demands are chosen to represent the full range of demand conditions that could be expected to occur in a network: High demand conditions represent the system at peak demand, whilst Low demand represents the system at minimum demand (here these demands are assumed to be $1.3 \mathrm{~kW}, 0.16 \mathrm{~kW}[34])$. A constraint violation is recorded in the LoVV and ECVV in (5), (7) irrespective of whether it only occurs at one of the High or Low demand conditions. This conservative approach means that if a violation is not recorded, that a network operator can conclude that there should never be a constraint violation. (This in contrast to approaches such as [10], which calculate a hosting capacity as a function of the hour of the day, for example.) The LoVV and ECVV should therefore be interpreted as there being potential for a constraint violation.

\section{LineAR MODELling APPROACH}

Linear network models are widely used in power systems [35]. In probabilistic power flow problems, they have the distinct advantage of speed and a broad range of analytic results [36], [37, Ch. 6]. The approach used in this work can be considered a hybrid approach [37, Ch. 6], with linearization used for fast Monte Carlo analysis for estimating the LoVV and ECVV. This is particularly appropriate as the many necessary load flow calculations [10] mean that computational efficiency is a key performance indicator.

A generic linearization of voltage magnitudes $|V|$ in real and reactive power injections $x$ can be denoted

$$
|V|=K x+b,
$$

where $K \in \mathbb{R}^{N_{V} \times N_{x}}$ denotes the linear sensitivity matrix, and $b$ is the offset vector. One linear model must be determined for each of the High demand and Low demand conditions (as described in Section II-B1). An approach for determining $K$ and $b$ for each of these models is therefore required.

Naturally, this choice of $K$ and $b$ will affect the accuracy of the linearisation (8) with respect to the non-linear load flow solution. There are two main approaches for determining the sensitivity matrix $K$ : those that calculate a power flow 
Jacobian exactly by calculating a Taylor expansion (e.g., the First Order Taylor method of [38]), and those that approximate the Jacobian by making use of some approximation-typically either assuming either assuming losses, load, or voltage drops are small [22], [35], [39], [40], or as one step of a fixedpoint iteration [38], [41]. The latter, approximate methods are often simpler to implement but will typically lead to increased linearization error compared to calculating the exact Jacobian. To improve the accuracy of load flow calculations, we therefore propose to use the voltage magnitude-power injection Jacobian as the sensitivity matrix $K$, with one model linearised for each of the Low and High demand conditions. The choice of the offset vector $b$ is discussed in Section III-B.

A further, specific problem which must be overcome for large systems, irrespective of the formulation, is that the memory requirements of $K$ can become very large, scaling with $N_{V}^{2}$ (e.g., the inverse of an admittance matrix of 50,000 nodes for [38] uses $40 \mathrm{~GB}$ of memory). Traditional Implicit methods form the sparse inverse of $K$ and then solve for $|V|$ [36], [40], although these methods can be relatively slow.

In this work we develop a novel, explicit linearization formulation based on two innovations, which are described in this section. The first is to represent $K$ using a novel factorization which we call the Block-Sparse-Plus-Low-Rank (BSLR) factorization, which simultaneously improves memory and computational (speed) performance. The second innovation partially updates the linearization, updating the offset $b$ to reduce the linearization error as the penetration $\rho$ is varied.

\section{A. BSLR Factorization}

The approach of this paper is to factorize (8) into $N_{N}$ coupled sub-problems, each having some dimension $N_{i}$, as

$$
\left|V_{i}\right|=K_{i} x_{i}+C_{i} F_{i} x+b_{i} \quad \forall i \in\left[1, N_{N}\right]
$$

where $\left|V_{i}\right|, K_{i}, x_{i}, b_{i}$ are partitions in the sub-problem dimension $N_{i}$, and $C_{i} \in \mathbb{R}^{N_{i} \times N_{C}}, F_{i} \in \mathbb{R}^{N_{C} \times N_{x}}$ are low-rank matrices, with the low rank coupling number $N_{C} \ll N_{x}$.

The factorization (9) is based on the observation that it is the (complex) MV voltage at the bus that an LV network is connected that can be thought of as being a point of common coupling from the LV to the MV circuit (see Fig. 1). A well-grounded three-phase system has three complex voltages associated with each bus and so there are six real-valued coupling variables (i.e., $N_{C}=6$ ). In other words, $F_{i}$ calculates the MV voltage at the top of sub-network $i$, as caused by the influence of all injections $x$; the impact on voltages from this MV node on LV voltages of circuit $i$ is then mapped through $C_{i}$; finally, voltage drops within cables in the LV circuit are then accounted for by the LV coupling matrix $K_{i}$.
Collecting terms, the model can be written

$$
\begin{aligned}
|V|=\left[\begin{array}{cccc}
K_{1} & 0 & \cdots & 0 \\
0 & K_{2} & \cdots & 0 \\
\vdots & \vdots & \ddots & \vdots \\
0 & 0 & \cdots & K_{n}
\end{array}\right] x \\
+\left[\begin{array}{cccc}
C_{1} & 0 & \cdots & 0 \\
0 & C_{2} & \cdots & 0 \\
\vdots & \vdots & \ddots & \vdots \\
0 & 0 & \cdots & C_{n}
\end{array}\right]\left[\begin{array}{c}
F_{1} \\
F_{2} \\
\vdots \\
F_{n}
\end{array}\right] x+b .
\end{aligned}
$$

A method for building the BSLR factorization is described in Appendix A.

There are parallels with the BSLR factorization (10) and the Hierarchical Distributed Algorithm decomposition that is used for low-level updates of Lagrangian variables in [22], necessarily based on the three-phase LinDistFlow formulation [42]. Specifically, the LinDistFlow formulation (based on a no-load, flat voltage solution [40]) means that the power injection from two nodes on the same network and same phase will result in identical voltage drops on branches outside of a given subnetwork. In contrast, the $F_{i}$ matrices of the BSLR factorization allow for the power-voltage sensitivity from each individual node to be modelled exactly, enabling a full factorization of the Jacobian. (This use of a Jacobian allows for significantly reduced power flow error compared to a flat voltage formulation, as we demonstrate in Section IV-A3.) Additionally, in contrast to the low-level abstraction of [22], the high-level matrix-matrix representation of the BSLR factorization is much more suited to efficient use of computational resources for fast power flow calculations, making use of highly optimized Basic Linear Algebra Subroutines (BLAS) implementations on modern processors [43, Ch. 1].

We note also that the BLSR factorization (10) is reminiscent of domain decomposition and diakoptic methods using in high performance computing applications (e.g., [44], [45]). In contrast to those works, however, the focus is on building a memory- and time-efficient, explicit linear model, rather than parallelization of the solution of non-linear equations.

1) Optimal Number of Partitions: To illustrate more concretely the behavior of the approach, we can consider the memory taken up by the factorization (9), relaxing the assumption that the network is partitioned by LV circuits, instead assuming that a method exists to partition network into uniform subnetworks of dimension $N_{N}$. For a given lowrank coupling number $N_{C}$, the amount of memory required $M_{\mathrm{Fczn}}$. (in bytes) to store the factorization $K_{i}, C_{i}, F_{i}$ for all $N_{N}$ systems is given by

$$
M_{\mathrm{Fczn} .}=m_{0} N_{N}\left(\frac{N_{V} N_{x}}{N_{N}^{2}}+N_{C}\left(\frac{N_{V}}{N_{N}}+N_{x}\right)\right),
$$

where $m_{0}$ is the number of bytes per entry. This is also directly proportional to the number of floating-point operations required for multiplications in (9).

Equation (11) highlights that not all factorizations perform equally in terms of memory and computation requirementsif there are too few partitions $N_{N}$ then the $K_{i}$ terms will 


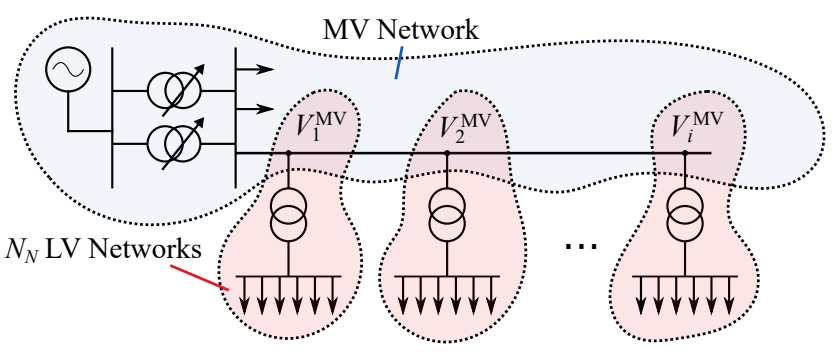

Fig. 1. The radial structure of distribution networks allows for computationally efficient representations of sensitivity matrices, with the BSLR factorization partitioning the MV-LV system into $N_{N} \mathrm{LV}$ networks, coupled by the MV system, as represented by (9).

dominate; if there are too many then the memory required for the $F_{i}$ terms will become larger than the original $K$ matrix. By differentiating and solving for $N_{N}$, it is found that the idealised optimal number of partitions $N_{N}^{\dagger}$ is given by

$$
N_{N}^{\dagger}\left(N_{V}\right)=\sqrt{\frac{N_{V}}{N_{C}}} .
$$

It is interesting to note that the optimal number of partitions $N_{N}^{\dagger}$ depends on the number of voltages $N_{V}$ to be calculated, but not the dimension of the input variable $N_{x}$.

2) Evaluating performance: To evaluate the performance the factorization (9), two pairs of metrics are calculated. The first pair of metrics are the time and memory Coefficient of Performances (COPs), $\eta_{\text {Mmry. }}, \eta_{\text {Mmry. }}$. These metrics compare the memory and speed of the 'Full' linearization (8) against the 'Factorized' linearization (9) (given subscripts $(\cdot)_{\text {Full }},(\cdot)_{\text {Fczn }}$ respectively) as

$$
\eta_{\text {Mmry. }}=\frac{M_{\text {Full }}}{M_{\text {Fczn }}}, \quad \eta_{\text {Time }}=\frac{t_{\text {Full }}}{t_{\text {Fczn }}},
$$

with the model memory requirement and calculation times of given matrix-matrix operations give denoted by $M_{(\cdot)}, t_{(\cdot)}$.

The final two metrics that are calculated are the theoretical optimal partitionings

$$
N_{P}^{\dagger}=N_{N}^{\dagger}\left(N_{P}\right), \quad N_{V}^{\dagger}=N_{N}^{\dagger}\left(2 N_{V}\right),
$$

calculated using (12), with $N_{P}^{\dagger}, N_{V}^{\dagger}$ denoting the optimal number of partitions considering calculations of voltage magnitudes at only $N_{P}$ loads, or real and imaginary voltages at $N_{V}$ nodes on a circuit.

These final two metrics are intended to provide an idea for the effectiveness of the proposed LV network partitioning (as discussed in Section (III-A)). Note that these are idealised, however, and it may not be possible to design an algorithm to partition a network into $N_{P}^{\dagger}, N_{V}^{\dagger}$ partitions (in fact, if the value of either of these metrics is not an integer then it is trivial to note that such a partitioning would not be possible). Instead, they are designed to indicate at a high-level if a given partitioning could have too many or too few partitions. The design of alternative partitioning schemes to the MV-LV scheme proposed here (which may be more efficient [46], [47], [42]) is beyond the scope of this work.

\section{B. Error Reduction with a Partial Linearization Update}

The second innovation in the linearization is to update the linearization offset vector $b$ whilst avoiding recalculating the BSLR model. The approach is similar to 'hot start' methods used for transmission system linearizations [35] or the fixedpoint method of [38].

In the first instance, we consider the calculation of a linearization using the First Order Taylor (FOT) method. In this approach, the inverse of the Jacobian of the power flow equations is found at a linearization point $x_{0}$, with the offset vector $b_{\mathrm{FOT}}$ given by

$$
b_{\mathrm{FOT}}=G\left(x_{0}\right)-K x_{0},
$$

where $G$ represents the mapping from injections to voltage magnitudes via the non-linear power flow equations.

Consider, however, if we know the mean of the power injections $\mu_{x}$. Then, we can decompose the power flow injections $x$ as

$$
x=x_{0}+\mu_{x}+\delta x,
$$

where $\delta x$ is the deviation about the mean. Then, we use a partial linear update (PLU), using an alternative offset vector $b_{\mathrm{PLU}}$ calculated as

$$
b_{\mathrm{PLU}}=G\left(x_{0}+\mu_{x}\right)-K x_{0}-K \mu_{x} .
$$

That is, a new value of $b_{\mathrm{PLU}}$ is calculated for a given value of the DER penetration $\rho$. (Note that the same sensitivity matrix $K$ is used in both (15) and (17).) Error analysis in Appendix $\mathrm{B}$ shows that, if the Jacobian is approximately constant, $b_{\mathrm{PLU}}$ will provide a better estimate than $b_{\mathrm{FOT}}$ at the cost of one non-linear load flow evaluation for each DER penetration $\rho$.

Finally, we also consider a Flat linear model [40] to highlight that the choice of sensitivity matrix $K$ can also have a significant effect on the linearization error. A sensitivity matrix $K_{\text {Flat }}$ is found at the no-load solution (i.e., with $x_{0}=0$ ); from this, the offset is then calculated in accordance with the PLU approach (17), i.e.,

$$
b_{\text {Flat }}=G\left(\mu_{x}\right)-K_{\text {Flat }} \mu_{x} .
$$

1) Error Metrics: To measure the error due to the linearization, we consider both the relative and absolute error in voltage deviations,

$$
\begin{aligned}
\epsilon_{\text {Rel. }} & =\frac{\left\|\left(G(x)-G\left(x_{0}\right)\right)-\left(\hat{G}(x)-G\left(x_{0}\right)\right)\right\|_{2}}{\left\|G(x)-G\left(x_{0}\right)\right\|_{2}}, \\
\epsilon_{\text {Abs. }} & =\left(G(x)-G\left(x_{0}\right)\right)-\left(\hat{G}(x)-G\left(x_{0}\right)\right),
\end{aligned}
$$

where $\hat{G}$ represents the linear load flow (9).

\section{Summary of Approach}

The steps for calculating LoVV and ECVV are as follows.

- The BSLR factorization of the sensitivity matrix $K$ is found at High and Low demand conditions (chosen as $1.3,0.16 \mathrm{kVA}$ at unity power factor, respectively [34]).

- For each DER penetration $\rho$, the linear offset vector $b_{\mathrm{PLU}}$ is calculated with the known injection $\mu_{x}$, 
TABLE I

INTEGRATED MV-LV CIRCUIT PARAMETERS. REAL LV NETWORK MODELS ARE ALLOCATED TO AGGREGATE MV NETWORK LOADS ACCORDING TO METHODS DESCRIBED IN [14].

\begin{tabular}{lllll}
\hline Ckt. ID & Power, MVA & No. Nodes & No. Lds. & No. LV Ckts. \\
\hline UG & 24.73 & 112,887 & 19,031 & 75 \\
UG/OH, A & 19.71 & 86,448 & 15,166 & 308 \\
UG/OH, B & 18.93 & 81,438 & 14,571 & 220 \\
OH, A & 9.15 & 37,032 & 7,048 & 354 \\
OH, B & 8.43 & 34,956 & 6,487 & 329 \\
OH/UG, A & 17.27 & 70,731 & 13,313 & 315 \\
OH/UG, B & 13.73 & 59,253 & 10,583 & 258 \\
\hline
\end{tabular}

- Voltages and voltage deviations are calculated for 3000 Monte Carlo runs at each of the High and Low demand conditions, at each DER penetration $\rho$.

- The plug-in-estimator enumerates the number of violations and number of affected customers, from which the LoVV and ECVV are calculated (in \%).

\section{RESUlts}

The goal of this paper is to study the impacts of frequency containment devices spread over a wide area. To capture the impacts of this geographic diversity, case studies are based on a set of seven MV-LV circuits, built by allocating real LV networks [48] to the aggregate loads of the UKGDS MV circuits [49], using the methods described in [14]. A summary of the circuit characteristics is given in Table I.

The rest of the section is structured as follows. We first consider the performance of the proposed BLSR factorization and PLU update in detail, to demonstrate their suitability for MV-LV probabilistic load flow required to understand the impacts of frequency response. Following this, calculations of the proposed risk indices are presented to evaluate the likelihood and potential severity of power quality impacts at LV caused by fast frequency response by domestic devices, addressing the central research gap identified in Section I. Finally, sensitivity analyses consider how changes in geographic dispersion of devices or DER power ratings could accentuate these risks, including a discussion of possible consequences should EVs be used to provide a fraction of the GB system frequency response requirement.

\section{A. Performance of Linearisation Approach}

First, we analyse the performance of the linearization approach described in Section III, to evaluate the performance of the BSLR factorization and ensure the partial linear update leads to acceptable error.

1) Memory COP: The first performance index of the matrix

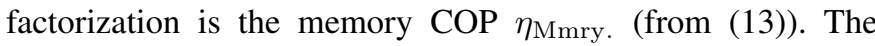
comparison for the seven circuits is given in Table II. The factorization leads to good performance, with the memory reduced by between 3 and 25 times.

This variation in performance is readily explained considering the number of partitions $N_{N}$ against the (idealised) optimal partition numbers $N_{V}^{\dagger}, N_{P}^{\dagger}$ (from (14)). In the UG circuit, the number of partitions lies between the optimal number for
TABLE II

THE MEMORY USED BY THE LINEAR MODELS $M_{\text {Fczn }}$. IS NOT TOO GREAT, ALTHOUGH THE MEMORY COP $\eta_{\text {Mmry. COULD LIKELY BE IMPROVED AS }}$ THE NUMBER OF PARTITIONS $N_{N}$ IS GREATER THAN THE OPTIMAL NUMBER OF PARTITIONS $N_{P}^{\dagger}, N_{V}^{\dagger}$ FOR SIX OF SEVEN CIRCUITS.

\begin{tabular}{lllll}
\hline Circuit ID & $\begin{array}{l}\text { Memory } \\
\text { requirement, } \\
M_{\text {Fczn. }}, \text { MB }\end{array}$ & $\begin{array}{l}\text { Memory } \\
\text { COP, } \\
\eta_{\text {Mmry. }}\end{array}$ & $\begin{array}{l}\text { No. } \\
\text { partitions, } \\
N_{N}\end{array}$ & $\begin{array}{l}\text { Opt. No. } \\
\text { Partitions, } \\
\left(N_{P}^{\dagger}, N_{V}^{\dagger}\right)\end{array}$ \\
\hline UG & 224.4 & 25.8 & 75 & $(56.3,194.0)$ \\
UG/OH, A & 483.5 & 7.6 & 308 & $(50.3,169.7)$ \\
UG/OH, B & 338.5 & 10.0 & 220 & $(49.3,164.8)$ \\
OH, A & 242.5 & 3.3 & 354 & $(34.3,111.1)$ \\
OH, B & 207.5 & 3.2 & 329 & $(32.9,107.9)$ \\
OH/UG, A & 419.7 & 6.8 & 315 & $(47.1,153.5)$ \\
OH/UG, B & 274.2 & 6.5 & 258 & $(42.0,140.5)$ \\
\hline
\end{tabular}

voltage and power calculations; however, all others have more partitions than either of these metrics.

2) Time COP: To calculate the time $\mathrm{COP} \eta_{\text {Time }}$, matrixmatrix calculations using the full sensitivity matrix and BSLR factorization are compared, as the number of columns in $x$ is varied (i.e., $x$ in (8) is chosen as a matrix with a varying number of columns). The median time of nine calculations is selected for determining the time COP (13).

The value of the time COP $\eta_{\text {Time }}$ is plotted in Fig. $2 \mathrm{a}$ as the number of columns increases from 10 to 10,000 . As the number of columns in $x$ increases, the time COP decreases, which is not unexpected as matrix-matrix operations are calculated using highly optimized subroutines. Nevertheless, there is a clear correlation between the theoretical speed up (given by the memory COP) and the COP seen in practice.

Additionally, we compare the time taken to calculate 3000 Monte Carlo runs using Implicit and BSLR methods in Fig. 2b (for the former, we form the sparse Jacobian in rectangular coordinates and then solve for $V$, as described in Section III). The BSLR method is significantly quicker than the Implicit method-a solution time of under ten seconds means that a user can interact with the program practically [50, Ch. 5.5]. The method also shows greater scalability-the largest network (the UG network) no longer has the slowest running time with the BSLR factorization (due to the more efficient factorization, as shown in Table II).

3) Error Analysis: The accuracy of the method is considered by calculating 100 load flow solutions, with a DER penetration of $10 \%$ using $3 \mathrm{~kW}$ devices. To consider the distribution of absolute error, the value of $\epsilon_{\mathrm{Abs}}$. is calculated at all customers across all 100 solutions. From this, the empirical distribution function (EDF) of the errors is calculated and plotted for each of the seven circuits in Fig. 3a. It is observed that more than $95 \%$ of errors are below $0.2 \%$ across all circuits. This behavior is commensurate with the performance of other linearizations, e.g., [41], [51].

Subsequently, we compare solutions using the partial linear update $b_{\mathrm{PLU}}$ against the FOT method using $b_{\mathrm{FOT}}$, as well as against the Flat model with $K_{\text {Flat }}, b_{\text {Flat }}$ (as described in Section III-B). The relative error $\epsilon_{\text {Rel }}$. is then calculated for each of the 100 solutions, and the distribution of relative errors for the three methods plotted in Fig. 3b. The PLU method with 


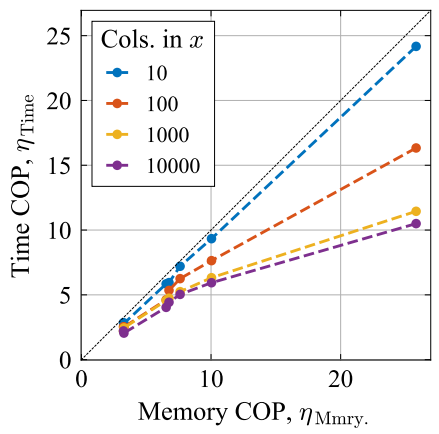

(a) Predicted and actual BSLR speedup

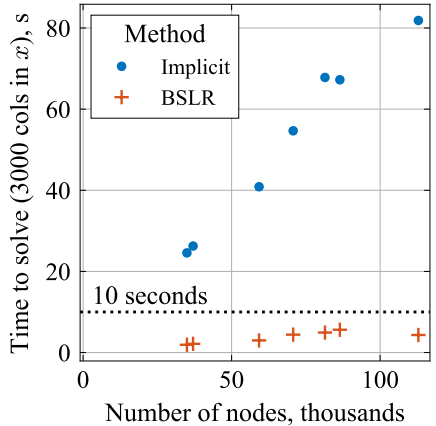

(b) BSLR and Implicit solution times

Fig. 2. Left: the BSLR theoretical memory and time coefficients of performance (COP) are equal, but larger matrix calculation subroutines tend to become more efficient, so the actual time COP is a function of the number of columns in the matrix $x$. Right: the BSLR factorization is an order of magnitude quicker than the Implicit approach.

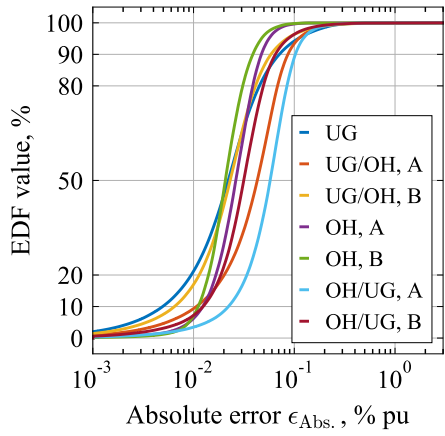

(a) Absolute error EDF

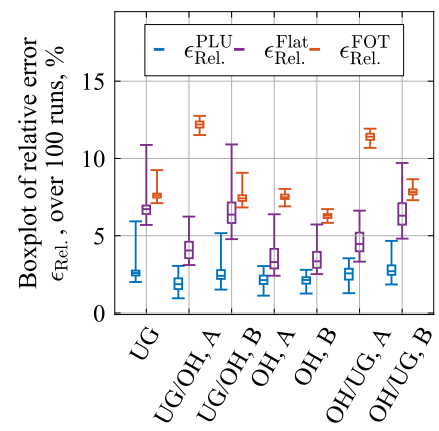

(b) Relative error range
Fig. 3. Load flow for 100 Monte Carlo runs are found (for $3 \mathrm{~kW}$ DERs at $10 \%$ penetration), from which (a) the EDF of the absolute errors $\epsilon_{\mathrm{Abs}}$. at all customers is plotted, as is (b) the range, interquartile range and median relative errors $\epsilon_{\text {Rel. }}$ as box plots (from (19), (20)), comparing the proposed PLU method against the Flat and FOT methods (as described in Section III-B).

the full Jacobian consistently leads to be smallest error. With respect to the FOT method, we see that the PLU maximum relative error is smaller than the smallest FOT relative error in all circuits. It also significantly outperforms the Flat method: across all networks and all simulations there was not a single simulation for which the relative error $\epsilon_{\text {Rel. }}$ of the Flat method (18) was less than that of the PLU method (17), and the median relative error $\epsilon_{\text {Rel. }}$ of the Flat model was between $55 \%$ and $164 \%$ greater than that of the proposed Jacobianbased approach.

\section{B. $M V-L V$ Stochastic Hosting Capacity}

The remainder of this section considers the LoVV and ECVV of the seven networks, with the goal of understanding when network constraint violations are most likely to become an issue. The values of these metrics are plotted for all seven circuits in Fig. 4 for the case in which devices increase loads by $3 \mathrm{~kW}$ (i.e., for high frequency containment). By considering the LoVV, it can be seen that the $20 \%$ SHC is reached between $1 \%$ and $10 \%$ penetration, depending on the circuit. On the other hand, the ECVV remains low, with a $1 \%$ ECVV value only above $4 \%$ penetration across all circuits. This implies

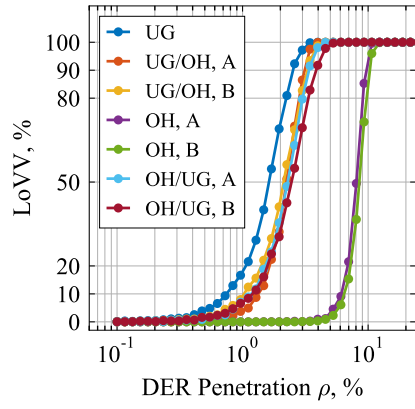

(a) LoVV for all circuits

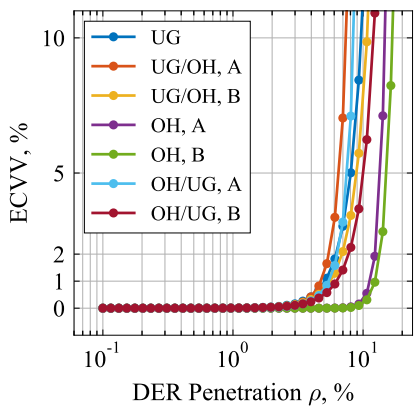

(b) ECVV for all circuits
Fig. 4. The LoVV and ECVV (from (5), (7)) for all seven of the circuits, with a load size $P_{0}$ of $3 \mathrm{~kW}$. The $20 \%$ SHC values are between $1 \%$ and $10 \%$ DER penetration, whilst the ECVV only crosses $1 \%$ above $4 \%$ DER penetration.

that constraint violations tend to remain local, even when a violation somewhere in the network becomes very likely.

It is notable that the $\mathrm{OH}$ circuits tend to have much lower LoVV values for a given DER penetration $\rho$. The MV models on which they are based have small secondary substation power demands. The LV network models allocated to those loads are therefore relatively small, which tend to have a much lower voltage drop than larger feeders [14].

The SHC values for all seven of the circuits are plotted as box plots in Fig. 5, in this instance considering increases in both loading and generation of $3 \mathrm{~kW}$. It is clear from this figure that SHC values are smaller for generation than for loading directions. To demonstrate why this is unsurprising, first consider that the UK statutory voltages limits are $(0.94$, 1.10) pu. For a voltage to drop to the lower voltage limit of $0.87 \mathrm{pu}$, if it was previously in this statutory range then voltage would have to drop by at least $0.07 \mathrm{pu}$. This is greater than the RVC constraint of $0.03 \mathrm{pu}$, and so only the RVC constraint is binding. On the other hand, under Low demand conditions, the voltages on the LV models are around 1.08 pu [14], and so the upper voltage limit of $1.10 \mathrm{pu}$ can be the binding constraint in those cases.

These simple observations illustrate that undervoltage constraints $V_{-}$are unlikely to be binding, whilst overvoltage constraints $V_{+}$could be binding. If low frequency containment is being considered, then more detailed analysis could find how these constraint violations change in time, compared to the (approximately) time-invariant RVC constraints.

\section{Sensitivity Analysis: Device Power Limits and Heteroge- neous LV Penetration}

The previous case studies assumed that devices will be $3 \mathrm{~kW}$, although EV fast-chargers of up to $32 \mathrm{~A}(7.4 \mathrm{~kW})$ can be installed in domestic properties in the UK. On the other hand, DER penetration is unlikely to be truly uniform-EVs are much more easily charged in neighbourhoods with offstreet parking, for example.

Given this, two sensitivity analyses are carried out on the UG circuit, considering high frequency containment (i.e. increasing the load). The first will consider how the device size impacts on the likelihood of violations. The second will focus on geographic diversity, considering how the results change 


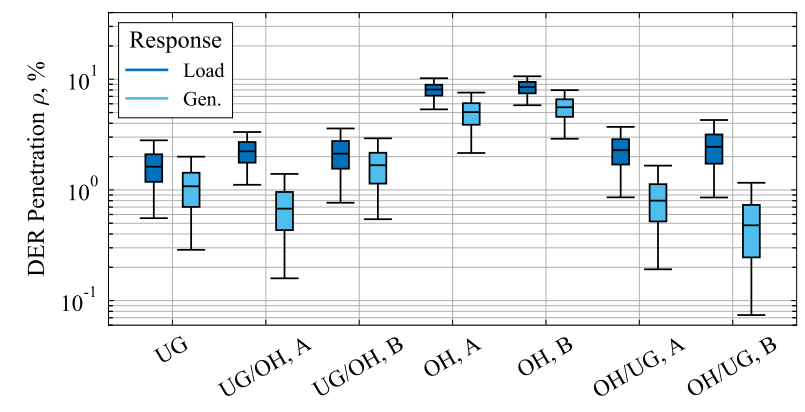

Fig. 5. The 5-, 25-, 50-, 75-, 95-\% SHC values (as defined in (6)) for the seven MV-LV circuits studied in this work. 'Load' frequency response is typically only limited by RVC constraints, where 'Generation' frequency response is limited by both RVC and overvoltage constraints.

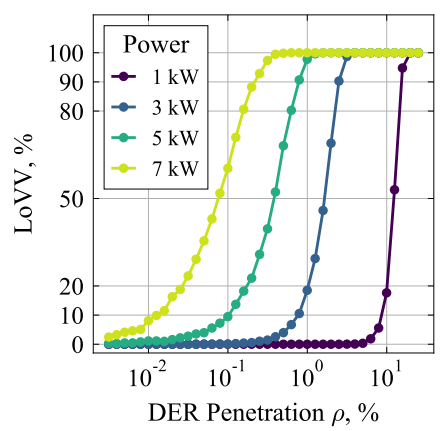

(a) LoVV against DER size

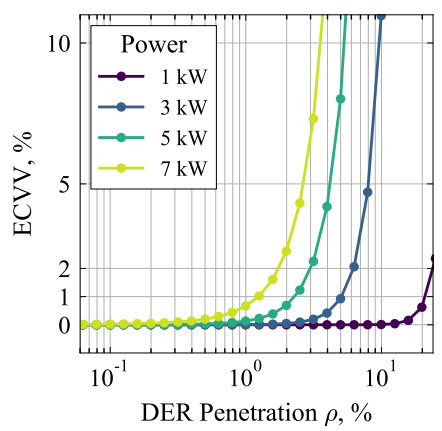

(b) ECVV against DER size
Fig. 6. The LoVV and ECVV against the device power $P_{0}$. The $10 \%$ SHC values for device sizes of $1,3,5,7 \mathrm{~kW}$ are $8.6,0.77,0.10,0.013 \%$ respectively.

if devices are much more likely to be taken up in specific neighbourhoods.

1) DER Sizing: Firstly, we consider the case with varying DER sizes, from $1 \mathrm{~kW}$ to $7 \mathrm{~kW}$. The LoVV and ECVV metrics are plotted against DER penetration $\rho$ in Fig. 6. From these figures it is clear that the LoVV (and thus SHC) values are strongly dependent on the sizing of the DERs. For example, for the $20 \%$ SHC value, approximately $10 \%$ of locations can have a $1 \mathrm{~kW}$ device, whilst the fraction is well under $0.1 \%$ for the $7 \mathrm{~kW}$ devices. The potential for power generation is thus much greater for devices of a smaller size. The ECVV is also dependent on the device size, with the ECVV increasing from $0.20 \%$ to $7.3 \%$ if the device size is increased from $3 \mathrm{~kW}$ to $7 \mathrm{~kW}$ at a DER penetration of $3 \%$.

In the context of the GB power system, this could severely limit the amount of response that should be allocated to domestic DERs without suitable distribution network management. For example, meeting one fifth (360 MW) of the GB loss of largest infeed requirements for 2022 [1] with $7 \mathrm{~kW} \mathrm{EV}$ chargers would be equivalent to DERs at $1.9 \%$ of 27 million domestic properties. The LoVV value in this case would be greater than $99 \%$, almost certainly leading to voltage violations (Fig. 6), with constraint violations expected to occur at more than $2 \%$ of properties.

2) DER Geographic Diversification: To consider how geographic diversification of DERs impacts the risk profile, it is assumed that DERs are allocated only to a subset of all LV networks within a circuit. Accordingly, the likelihood of

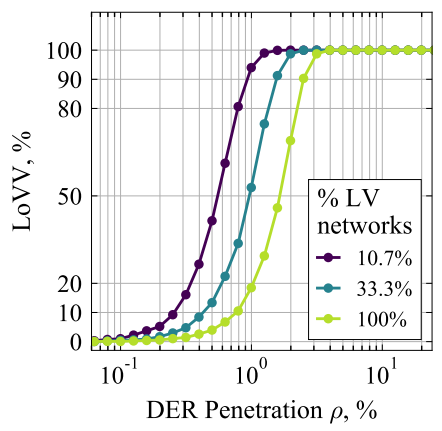

(a) LoVV against \% LV Networks

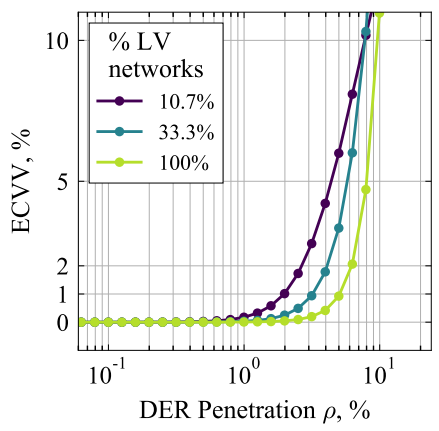

(b) ECVV against \% LV Networks
Fig. 7. The LoVV and ECVV plotted against the \% LV Networks (i.e., the fraction of LV networks to which DERs can be allocated). The $10 \%$ SHC value decreases from $0.77 \%$ to $0.43 \%, 0.26 \%$ if $33.3 \%$ or $10.7 \%$ of LV feeders have frequency responsive devices, respectively.

a DER being allocated in those networks is then increased in proportion to the fraction of LV networks allocated. ${ }^{1}$

The ECVV and LoVV for these cases are plotted in Fig. 7. It is interesting to see that the diversification does not make as stark a difference as with the DER size (comparing, for example, $1,3 \mathrm{~kW}$ cases against $33.3 \%, 100 \%$ diversification). Nevertheless, there a noticeable increase in the LoVV and ECVV, with the ECVV at a DER penetration of 3\% increasing from $0.20 \%$ to $2.78 \%$ as the diversification (no. LV feeders) reduces from $100 \%$ to $10.7 \%$.

\section{CONCLUSION}

This paper proposes a novel approach for studying the impacts of frequency containment services, provided by domestic DERs, on the likelihood and severity of network constraint violations. The BSLR factorization allows for computationally efficient linearizations of large network models, increasing calculation speeds by as much as 20 times without compromising model fidelity. The use of a partial linear update reduces the median relative error by more than half in all circuits studied; furthermore, it is shown that models based on a no-load linearization have $50 \%$ greater median relative error than the proposed Jacobian-based linearization. The risk of violations is shown to be strongly dependent on the DER sizes and geographic spread-case studies show small $1 \mathrm{~kW}$ devices spread throughout networks are very unlikely to ever cause constraint violations. Rapid voltage change constraints are critical-the risk of overvoltage violations varies in time, whilst undervoltages have been demonstrated to never be binding in reasonable cases.

The provision of flexibility for TSO services could result in much greater demand-side geographic and temporal coupling, requiring DSOs and aggregators to greatly expand the reach of active network management tools. We conclude that tractable, practical and efficient methods for analyzing and managing

\footnotetext{
${ }^{1}$ For example, if we are studying the UG network with $10.7 \%$ diversification, then 8 of the $75 \mathrm{LV}$ networks are chosen (at random) for allocation; the likelihood of a DER being allocated is increased by a factor of $0.107^{-1}$. The mean power of the $10.7 \% \mathrm{LV}$ Network case at a given DER penetration $\rho$ is therefore identical to the full (100\%) case with the same DER penetration.
} 
large-scale network models will therefore become indispensable tools for distribution networks as they transition to net zero.

\section{ACKNOWLEDGMENT}

This work was funded by the EPSRC through grant no. EP/S00078X/1 (Supergen Energy Networks Hub 2018). The authors are grateful for fruitful discussions with Sasa Djokic on power quality and Filiberto Fele on matrix factorizations.

\section{APPENDIX A: BSLR FACTORIZATION}

The goal is to calculate the BSLR linearization (9). We use the notation

$$
\partial_{x} f=\left[\begin{array}{c}
\partial_{x}^{\mathfrak{R}} f \\
\partial_{x}^{\mathfrak{J}} f
\end{array}\right], \quad X^{\boxplus}=\left[\begin{array}{cc}
X^{\mathfrak{R}} & -X^{\mathfrak{I}} \\
X^{\mathfrak{I}} & X^{\mathfrak{R}}
\end{array}\right], \quad\langle x\rangle=\operatorname{diag}(x),
$$

where $\partial_{x}^{\Re} f, \partial_{x}^{\mathfrak{I}} f$ denotes the taking of the partial derivative of the real or imaginary part of function $f$ with respect to $x$, respectively, $\bar{x}$ denotes the complex conjugate of $x$, and $X^{\mathfrak{R}}, X^{\mathfrak{I}}$ are the real and imaginary parts of $X$.

The power flow equations for the $i$-th, wye-connected LV network (as in Fig. 1) can be written [41]

$$
\begin{aligned}
S_{i}^{\mathrm{LV}} & =\left\langle V_{i}^{\mathrm{LV}}\right\rangle \bar{I}_{i}^{\mathrm{LV}}, \\
I_{i}^{\mathrm{LV}} & =Y_{i}^{\mathrm{MV}: \mathrm{LV}} V_{i}^{\mathrm{MV}}+Y_{i}^{\mathrm{LV}: \mathrm{LV}} V_{i}^{\mathrm{LV}},
\end{aligned}
$$

where $S_{i}^{\mathrm{LV}}$ are the complex power injections, $V_{i}^{\mathrm{LV}}$ are the complex voltages, $I_{i}^{\mathrm{LV}}$ are the complex power injections, and the admittance matrix is partitioned suitably into $Y_{i}^{\mathrm{MV}: \mathrm{LV}}, Y_{i}^{\mathrm{LV}: \mathrm{LV}}$ as in [41].

Assume that the partial derivative $\partial_{x} V_{i}^{\mathrm{MV}}$ known. Taking partial derivatives of the power flow equations yields

$$
\begin{aligned}
\left\langle V_{i}^{\mathrm{LV} \boxplus}\right\rangle\left(\bar{Y}_{i}^{\mathrm{MV}: \mathrm{LV} \boxplus} \partial_{x} \bar{V}_{i}^{\mathrm{MV}}+\bar{Y}_{i}^{\mathrm{LV}: \mathrm{LV} \boxplus} \partial_{x} \bar{V}_{i}^{\mathrm{LV}}\right) \\
+\left\langle\bar{I}_{i}^{\mathrm{LV} \boxplus}\right\rangle \partial_{x} V_{i}^{\mathrm{LV}}=\partial_{x} S_{i}^{\mathrm{LV}},
\end{aligned}
$$

where $(\cdot)^{\boxplus},\langle\cdot\rangle$ are defined as in (21). By collecting common factors, these can be written equivalently as

$$
A \partial_{x} V_{i}^{\mathrm{LV}}=\partial_{x} S_{i}^{\mathrm{LV}}+B \partial_{x} \bar{V}_{i}^{\mathrm{MV}} .
$$

Written in this form, we write down the BSLR formulation (in complex voltages)

$$
\hat{K}_{i}=A^{-1} \partial_{x} S_{i}^{\mathrm{LV}}, \quad \hat{C}_{i}=A^{-1} B, \quad \hat{F}_{i}=\partial_{x} \bar{V}_{i}^{\mathrm{MV}} .
$$

To see this, note that, for bus injections not in the $i$ th LV network, $x_{\backslash i}$, the partial derivative $\partial_{x_{\backslash i}} S_{i}^{\mathrm{LV}}$ is zero, and so $\hat{K}_{i}$ is zeros except for those buses in the $i$ th LV network. Crucially, note that $\partial_{x} \bar{V}_{i}^{\mathrm{MV}} \in \mathbb{R}^{6 \times N_{x}}$, which is a 'wide' matrix, whilst $B \in \mathbb{R}^{2 N_{i}^{\mathrm{LV}} \times 6}$ is a 'tall' matrix. (The dimension is six in these matrices as there are three coupling voltage phasors.)

The final steps therefore are to calculate $\partial_{x} \bar{V}_{i}^{\mathrm{MV}}$ and the matrices in voltage magnitudes. The former is found by partitioning the partial derivatives of the MV-LV network into LV and MV buses (formed from power flow equations as (22), but indexes updated appropriately), then solving via Schur's complement (see, e.g., [47, Ch. 14.2]). The model in voltage magnitudes can be found using [38]

$$
\frac{\partial|f|}{\partial x}=\Re\left(\frac{\bar{f}}{|f|} \frac{\partial f}{\partial x}\right)
$$

to calculate $K_{i}, C_{i}, F_{i}$ from (25) as required.

\section{APPENDiX B: PARTIAL Linear UPDATE ERROR ANALYSiS}

For a Jacobian matrix of the system of equations $G$ linearised at $x_{0}, J_{x_{0}}$, we can write down:

$G\left(x_{0}+\mu_{x}+\delta x\right)=G\left(x_{0}\right)+J_{x_{0}}\left(\mu_{x}+\delta x\right)+\mathcal{O}_{G}\left(\left\|\mu_{x}+\delta x\right\|^{2}\right)$,

where $\mathcal{O}_{(\cdot)}(\alpha)$ denotes an error of the order $\alpha$.

If the linearization around $x_{0}+\mu_{x}$ were known, then the error term in (27) could be reduced to only be $\mathcal{O}_{G}\left(\|\delta x\|^{2}\right)$. It is expensive to calculate the Jacobian $J_{x_{0}+\mu_{x}}$ at the new point $x_{0}+\mu_{x}$; on the other hand, one function evaluation $G\left(x_{0}+\mu_{x}\right)$ is not. The error in the Jacobian is

$$
J_{x_{0}+\mu_{x}}=J_{x_{0}}+\mathcal{O}_{J}\left(\left\|\mu_{x}\right\|\right),
$$

so (27) can instead be calculated as

$$
\begin{aligned}
G\left(x_{0}+\mu_{x}+\right. & \delta x)=G\left(x_{0}+\mu_{x}\right) \\
& +\left(J_{x_{0}}+\mathcal{O}_{J}\left(\left\|\mu_{x}\right\|\right)\right) \delta x+\mathcal{O}_{G}\left(\|\delta x\|^{2}\right) .
\end{aligned}
$$

Comparing (27), (29), the error $\mathcal{O}_{G}\left(\left\|\mu_{x}+\delta x\right\|^{2}\right)$ has been reduced to $\mathcal{O}_{J}\left(\left\|\mu_{x}\right\|\right) \times \mathcal{O}_{G}(\|\delta x\|)+\mathcal{O}_{G}\left(\|\delta x\|^{2}\right)$. So long as $\mathcal{O}_{J} \approx \mathcal{O}_{G}$ (i.e., the Jacobian does not change too fast), the error will therefore be reduced.

\section{REFERENCES}

[1] V. Levi, G. Williamson, J. King, and V. Terzija, "Development of GB distribution networks with low carbon technologies and smart solutions: Scenarios and results," International Journal of Electrical Power \& Energy Systems, vol. 119, p. 105832, 2020.

[2] British Standards Institute, "PAS 1878: Classification of energy smart PAS 1878:2021," https://standardsdevelopment.bsigroup.com/projects/ 2019-01576, 2020.

[3] J. Bialek, "What does the power outage on 9 August 2019 tell us about GB power system," in Cambridge Working Papers in Economics: 2018, 2020 .

[4] K. Pandiaraj, P. Taylor, N. Jenkins, and C. Robb, "Distributed load control of autonomous renewable energy systems," IEEE Transactions on Energy Conversion, vol. 16, no. 1, pp. 14-19, 2001.

[5] M. T. Muhssin, L. M. Cipcigan, N. Jenkins, S. Slater, M. Cheng, and Z. A. Obaid, "Dynamic frequency response from controlled domestic heat pumps," IEEE Transactions on Power Systems, vol. 33, no. 5, pp. 4948-4957, 2018.

[6] Y. Mu, J. Wu, J. Ekanayake, N. Jenkins, and H. Jia, "Primary frequency response from electric vehicles in the Great Britain power system," IEEE Transactions on Smart Grid, vol. 4, no. 2, pp. 1142-1150, 2012.

[7] T. Clarke, T. Slay, C. Eustis, and R. B. Bass, "Aggregation of residential water heaters for peak shifting and frequency response services," IEEE Open Access Journal of Power and Energy, vol. 7, pp. 22-30, 2019.

[8] V. Trovato, I. M. Sanz, B. Chaudhuri, and G. Strbac, "Advanced control of thermostatic loads for rapid frequency response in Great Britain," IEEE Transactions on Power Systems, vol. 32, no. 3, pp. 2106-2117, 2016.

[9] S. Taheri, M. Jalali, V. Kekatos, and L. Tong, "Fast probabilistic hosting capacity analysis for active distribution systems," IEEE Transactions on Smart Grid, 2020.

[10] A. Dubey and S. Santoso, "On estimation and sensitivity analysis of distribution circuit's photovoltaic hosting capacity," IEEE Transactions on Power Systems, vol. 32, no. 4, pp. 2779-2789, 2016. 
[11] J. Smith and M. Rylander, "Stochastic analysis to determine feeder hosting capacity for distributed solar PV," Electric Power Research Inst., Palo Alto, CA, Tech. Rep, vol. 1026640, pp. 0885-8950, 2012.

[12] H. Al-Saadi, R. Zivanovic, and S. F. Al-Sarawi, "Probabilistic hosting capacity for active distribution networks," IEEE Transactions on Industrial Informatics, vol. 13, no. 5, pp. 2519-2532, 2017.

[13] A. G. Givisiez, K. Petrou, and L. F. Ochoa, "A review on tso-dso coordination models and solution techniques," Electric Power Systems Research, vol. 189, p. 106659, 2020.

[14] M. Deakin, D. Greenwood, S. Walker, and P. Taylor, "Hybrid European MV - LV network models for smart distribution network modelling," https://arxiv.org/abs/2009.14240, 2020.

[15] L. Gutierrez-Lagos and L. F. Ochoa, "Opf-based cvr operation in pvrich mv-lv distribution networks," IEEE Transactions on Power Systems, vol. 34, no. 4, pp. 2778-2789, 2019.

[16] A. T. Procopiou, K. Petrou, L. F. Ochoa, T. Langstaff, and J. Theunissen, "Adaptive decentralized control of residential storage in pv-rich mv-lv networks," IEEE Transactions on Power Systems, vol. 34, no. 3, pp 2378-2389, 2018.

[17] National Grid Electricity Transmission, "Frequency sensitive electric vehicle and heat pump power consumption (closedown report)," https://www.smarternetworks.org/project/nia_nget0138, 2016, accessed $15 / 9 / 20$.

[18] S. C. Ross, G. Vuylsteke, and J. L. Mathieu, "Effects of load-based frequency regulation on distribution network operation," IEEE Transactions on Power Systems, vol. 34, no. 2, pp. 1569-1578, 2018.

[19] S. C. Ross and J. L. Mathieu, "Strategies for network-safe load control with a third-party aggregator and a distribution operator," IEEE Transactions on Power Systems, 2021.

[20] E. Vrettos and G. Andersson, "Combined load frequency control and active distribution network management with thermostatically controlled loads," in 2013 IEEE International Conference on Smart Grid Communications (SmartGridComm). IEEE, 2013, pp. 247-252.

[21] I. Jendoubi, K. Sheshyekani, and H. Dagdougui, "Aggregation and optimal management of tcls for frequency and voltage control of a microgrid," IEEE Transactions on Power Delivery, 2020.

[22] X. Zhou, Z. Liu, C. Zhao, and L. Chen, "Accelerated voltage regulation in multi-phase distribution networks based on hierarchical distributed algorithm," IEEE Transactions on Power Systems, vol. 35, no. 3, pp. 2047-2058, 2019

[23] E. Dall'Anese, S. S. Guggilam, A. Simonetto, Y. C. Chen, and S. V. Dhople, "Optimal regulation of virtual power plants," IEEE Transactions on Power Systems, vol. 33, no. 2, pp. 1868-1881, 2017.

[24] N. Grid, "Future frequency response products: Faster active response," https://www.nationalgrideso.com/document/119256/download, 2018, accessed 9th Sept 2020

[25] R. C. Dugan, M. F. McGranaghan, and H. W. Beaty, Electrical power systems quality, 2nd ed. McGraw-Hill, 2002.

[26] C. P. Gupta and J. V. Milanovic, "Probabilistic assessment of equipment trips due to voltage sags," IEEE Transactions on Power Delivery, vol. 21, no. 2, pp. 711-718, 2006

[27] D. M. Greenwood and P. C. Taylor, "Unlocking the benefits of realtime thermal ratings through probabilistic power network planning," IET Generation, Transmission \& Distribution, vol. 8, no. 12, pp. 2055-2064, 2014.

[28] UK Parliament, "The electricity safety, quality and continuity regulations $2002, " 2002$.

[29] ENA Task Group on Statutory Voltage Limits, "Statutory voltage limits at customers' terminals in the UK and options for future application of wider limits at low voltage," Energy Networks Association, Tech. Rep., 2017.

[30] Operations Directorate of the ENA, "Engineering recommendation P28, issue 2: Voltage fluctuations and the connection of disturbing equipment to transmissions systems and distribution networks in the United Kingdom,” Energy Networks Association, Tech. Rep., 2017.

[31] A. Dubey, S. Santoso, and A. Maitra, "Understanding photovoltaic hosting capacity of distribution circuits," in 2015 IEEE power \& energy society general meeting. IEEE, 2015.

[32] A. Brooks, E. Lu, D. Reicher, C. Spirakis, and B. Weihl, "Demand dispatch," IEEE Power and Energy Magazine, vol. 8, no. 3, pp. 20-29, 2010.

[33] L. Wasserman, All of statistics: a concise course in statistical inference. Springer Science \& Business Media, 2013.

[34] S. Ingram, S. Probert, and K. Jackson, "The impact of small scale embedded generation on the operating parameters of distribution networks," PB Power, Department of Trade and Industry (DTI), 2003.
[35] B. Stott, J. Jardim, and O. Alsaç, "DC power flow revisited," IEEE Transactions on Power Systems, vol. 24, no. 3, pp. 1290-1300, 2009.

[36] B. R. Prusty and D. Jena, "A critical review on probabilistic load flow studies in uncertainty constrained power systems with photovoltaic generation and a new approach," Renewable and Sustainable Energy Reviews, vol. 69, pp. 1286-1302, 2017.

[37] B. W. Tuinema, J. L. R. Torres, A. I. Stefanov, F. M. Gonzalez-Longatt, and M. A. van der Meijden, Probabilistic Reliability Analysis of Power Systems. Springer, 2020.

[38] A. Bernstein, C. Wang, E. Dall'Anese, J.-Y. Le Boudec, and C. Zhao, "Load flow in multiphase distribution networks: Existence, uniqueness, non-singularity and linear models," IEEE Transactions on Power Systems, vol. 33, no. 6, pp. 5832-5843, 2018.

[39] A. Bernstein and E. Dall'Anese, "Linear power-flow models in multiphase distribution networks," in 2017 IEEE PES Innovative Smart Grid Technologies Conference Europe (ISGT-Europe). IEEE, 2017.

[40] S. Bolognani and F. Dörfler, "Fast power system analysis via implicit linearization of the power flow manifold," in 2015 53rd Annual Allerton Conference on Communication, Control, and Computing (Allerton). IEEE, 2015, pp. 402-409.

[41] S. Bolognani and S. Zampieri, "On the existence and linear approximation of the power flow solution in power distribution networks," IEEE Transactions on Power Systems, vol. 31, no. 1, pp. 163-172, 2015.

[42] X. Zhou, Y. Chen, Z. Liu, C. Zhao, and L. Chen, "Multi-level optimal power flow solver in large distribution networks," in 2020 IEEE International Conference on Communications, Control, and Computing Technologies for Smart Grids (SmartGridComm). IEEE, 2020, pp. 1-6.

[43] G. H. Golub and C. F. Van Loan, Matrix computations. JHU press, 2012.

[44] P. Aristidou, S. Lebeau, and T. Van Cutsem, "Power system dynamic simulations using a parallel two-level Schur-complement decomposition," IEEE Transactions on Power Systems, vol. 31, no. 5, pp. 39843995,2015

[45] T. Xiao, W. Tong, and J. Wang, "Study on reducing the parallel overhead of the BBDF method for power system transient stability simulations," IEEE Transactions on Power Systems, vol. 35, no. 1, pp. 539-550, 2019

[46] K. Chan, R. Dunn, and A. Daniels, "Efficient heuristic partitioning algorithm for parallel processing of large power systems network equations,' IEE Proceedings-Generation, Transmission and Distribution, vol. 142, no. 6, pp. 625-630, 1995

[47] Y. Saad, Iterative methods for sparse linear systems. SIAM, 2003.

[48] Electricity Northwest (ENWL), "Low voltage network solutions: LV network models," https://www.enwl.co.uk/innovation/smaller-projects/ low-carbon-networks-fund/low-voltage-network-solutions/, 2015.

[49] C. Foote, P. Djapic, G. Ault, J. Mutale, and G. Strbac, "United kingdom generic distribution system (ukgds): Defining the generic networks," https://github.com/sedg/ukgds, October 2005.

[50] J. Nielsen, Usability engineering. Morgan Kaufmann, 1994

[51] A. Gonzalez, F. M. Echavarren, L. Rouco, and T. Gomez, "A sensitivities computation method for reconfiguration of radial networks," IEEE Transactions on Power Systems, vol. 27, no. 3, pp. 1294-1301, 2012. 